ACCEPTED MANUSCRIPT

\title{
Enhanced chemical etch rate of borosilicate glass via spatially resolved laser-generated color centers
}

To cite this article before publication: Anton Serkov et al 2019 J. Phys. D: Appl. Phys. in press https://doi.org/10.1088/1361-6463/ab6515

\section{Manuscript version: Accepted Manuscript}

Accepted Manuscript is "the version of the article accepted for publication including all changes made as a result of the peer review process, and which may also include the addition to the article by IOP Publishing of a header, an article ID, a cover sheet and/or an 'Accepted

Manuscript' watermark, but excluding any other editing, typesetting or other changes made by IOP Publishing and/or its licensors"

This Accepted Manuscript is @ 2019 IOP Publishing Ltd.

During the embargo period (the 12 month period from the publication of the Version of Record of this article), the Accepted Manuscript is fully protected by copyright and cannot be reused or reposted elsewhere.

As the Version of Record of this article is going to be / has been published on a subscription basis, this Accepted Manuscript is available for reuse under a CC BY-NC-ND 3.0 licence after the 12 month embargo period.

After the embargo period, everyone is permitted to use copy and redistribute this article for non-commercial purposes only, provided that they adhere to all the terms of the licence https://creativecommons.org/licences/by-nc-nd/3.0

Although reasonable endeavours have been taken to obtain all necessary permissions from third parties to include their copyrighted content within this article, their full citation and copyright line may not be present in this Accepted Manuscript version. Before using any content from this article, please refer to the Version of Record on IOPscience once published for full citation and copyright details, as permissions will likely be required. All third party content is fully copyright protected, unless specifically stated otherwise in the figure caption in the Version of Record.

View the article online for updates and enhancements. 


\title{
Enhanced chemical etch rate of borosilicate glass via spatially resolved laser-generated color centers
}

\author{
A. A. Serkov, H. V. Snelling \\ Department of Physics and Mathematics, Faculty of Science and Engineering, University of \\ Hull, HU6 7RX, Hull, United Kingdom
}

\begin{abstract}
In this work, it is shown that controllable increases in chemical reactivity of borosilicate glass can be induced through spatially resolved femtosecond laser irradiation at fluence values significantly lower than the damage threshold. The hydrofluoric acid etch rate has been found to be closely correlated to the reduction in optical transmission of the glass at $488 \mathrm{~nm}$, which is, in turn, governed by the production of boron-oxygen hole centers. The combination of laser irradiation below the ablation threshold followed by chemical etching is shown to yield surfaces that have a roughness lower than those achieved by either laser or chemical etching alone. Application of this effect to the manufacture of freeform Laplacian optics is demonstrated.
\end{abstract}

\section{Introduction}

Developments in freeform optical science has led to inyestigations into new manufacturing techniques. Conventional machining methods, such as precise CNC milling or cutting with the attendant need for polishing [1] are typically rather expensive and time consuming. One of the possible ways to overcome this problem is to employ laser micromachining. The most straightforward way to do that is direct laser ablation of glasses. Excimer laser machining utilising the large intrinsic optical absorption in the UV has shown promise [2,3] as well as laser-induced non-linear absorption via ultrashort pulses [4-7]. The longer irradiation timescale of carbon dioxide lasers have allowed melt flow to occur and a degree of polishing to be accomplished $[8,9]$.

An elegant way to induce laser absorption in glass while still using nanosecond laser pulses of relatively low photon energies was proposed in [10]. The main idea of the method consists in irradiation of the glass sample whilst it is situated in contact with an organic liquid that has a high absorption coefficient at the wavelength of incident laser radiation. The glass surface is thus indirectly processed either by plasma plume or by the cavitation bubbles forming in the liquid. This method has become quite popular since then, as it can be implemented using commercially available nanosecond laser sources of UV [11-14], visible [15,16], or even NIR [17] wavelengths. However, because of the low repetition rate of most excimer lasers (typically $10-200 \mathrm{~Hz}$, and up to $2 \mathrm{kHz}$ in specialist devices), this method cannot be easily scaled in the UV. Furthermore, the method is sensitive to the laser parameters, and thus, for precise micromachining, additional feedback control of the laser output would be required.

The most promising candidate to fabricate the freeform optics among the laser-assisted techniques is the so-called "femtosecond laser-assisted chemical etching" (FLICE), which was first proposed in [18] to create microchannels in fused silica. This method requires femtosecond laser irradiation of sufficient intensity to alter the chemical properties of the glass. It should be noted that the main application of this method is for microfluidics [19-21], 
where optical quality of the final surface is not normally required. The initial femtosecond irradiation is thus typically carried out at fluence values exceeding the damage threshold, which provides higher etching rates accompanied by increased roughness. However, FLICE has been used for optical applications, such as for micro-lens [22] and prism array fabrication [23].

A variation of the FLICE method was proposed for silver ion-exchanged glasses [24,25], and later for cerium ion-exchanged glass (Foturan) [26]. In these works, due to the intrinsic properties of the glasses used, the authors could use laser sources of relatively low power to change the etching rate. For instance, in $[24,25]$ the authors used a CW laser with an average power of less than $5 \mathrm{~mW}$, which is comparable to that of a commercially available laser pointer. Even though both examples seem promising for freeform optics fabrication, their main disadvantage is either the cost (in case of Foturan), or the availability (in case of HEBS) of the material used.

In the case of ion-exchanged glasses, the mechanism of the reactivity change is quite clear as selective crystallization of the target material occurs. However, similar effects were observed for other types of glass [27-29] not having any additional ions in their structure that might serve as crystallization centers. The authors of [27] found the change in reactivity to be dependent on the presence of boron in the glass composition and in [28] showed the correlation between the etching depth and the electron spin resonance peak intensity of boron-related color centers.

In this work, the interaction of femtosecond laser pulses with borosilicate glass (Schott Borofloat 33 [30]) is investigated. The effect of laser irradiation below the damage threshold of the glass is found to darken the material (an increase in optical density) and is attributed to the formation of boron-oxygen hole centers. Such defects in glasses are often referred to as color centers due to the changes in the optical transmission spectrum associated with them (historically see [31-33]). It is found that this darkening correlates to an increase in acid reactivity and so anisotropic chemical etching can be induced in a controlled manner. An application of this process to the fabrication of freeform optics is demonstrated.

\section{Experimental setup}

In all the color center generation experiments a Ti:Sapphire (Spectra-Physics Hurricane) laser was used as the radiation source. The parameters were as follows: wavelength of $800 \mathrm{~nm}$, repetition rate of $1 \mathrm{kHz}$, maximum average power of $600 \mathrm{~mW}$, and a typical pulse duration of $140 \mathrm{fs}$. A fused silica plano-convex lens with a diameter of $25.4 \mathrm{~mm}$ and a $100 \mathrm{~mm}$ focal length was used to focus the laser beam. Aerotech ALS-130 translation stages were used to move the sample during the irradiation process. The scanning speed was adjusted to achieve the desired number of pulses per point.

Borofloat 33 glass with the thickness values of 1,2,3, and $5 \mathrm{~mm}$ was used as a target material in all the HF etching experiments.

The etching process was carried out using a 10\% HF solution and lasted for 1 hour in an ultrasonic bath at room temperature. The surfaces of the samples were measured and analysed by stylus profilometry (Bruker Dektak XT), and white-light interferometry (Wyko NT1100). A Zeiss Evo 60 scanning electron microscope was also used to visualise the surface of the etched samples. 
Various techniques have been utilised to study color centers in glass, for example Electron Paramagnetic Resonance (EPR) and Electron Spin Resonance (ESR) [34,35]. In this work, spatially resolved studies were required and so optical techniques were favoured. In particular, optical absorption measurements were implemented. Initially, spatially averaged transmission spectra were recorded using a Horiba Fluoromax spectrometer to find the main absorption features so as to choose an appropriate wavelength probe for spatially resolved scanning. Samples were prepared by femtosecond laser irradiation incident on the narrow side of a $25 \times 25 \times 3 \mathrm{~mm}$ Borofloat 33 flat plate. The irradiation geometry is schematically shown in Fig.1.

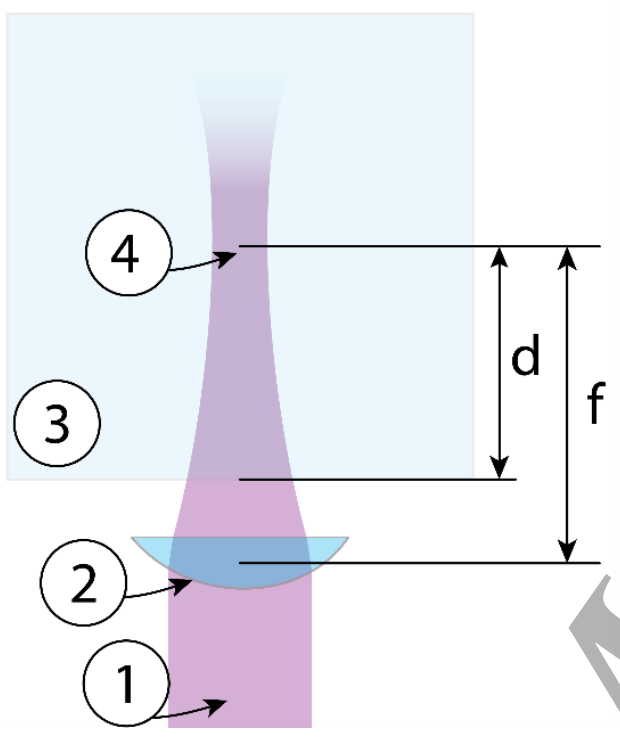

a

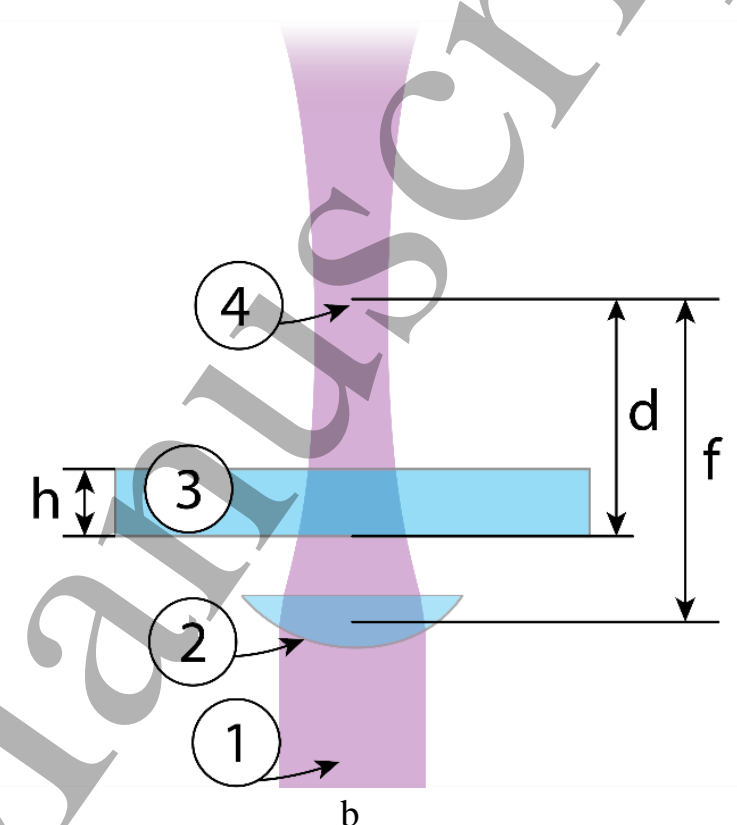

b

Figure 1. Two geometries were utilised in the laser exposure experiments. In (a) the irradiation scheme to generate color centers for subsequent optical density mapping is shown. Samples that were

chemically etched were thinner (b) and so the beam exit surface could be observed to show an increase in etch rate. Key: (1) Laser beam, (2) focussing lens, (3) borosilicate glass sample, (4) geometric focus location, (f) focal length of the lens, (d) distance between the front surface of the sample and the geometric focus, (h) sample thickness

The cross-section of the color centers formed in this way could then be visualized by observing the darkening of the glass when viewed through the $25 \times 25 \mathrm{~mm}$ face. Point-bypoint mapping of the transmission change was performed by measuring the transmission cross-sections at different values of propagation depth (step size of $50 \mu \mathrm{m}$ ) and concatenating the resulting data. Light from a Thorlabs OSL-2 lamp, spectrally narrowed using a Thorlabs FL488-10 bandpass filter (center wavelength of $488 \mathrm{~nm}$, with $10 \mathrm{~nm}$ FWHM), was focused by a cylindrical lens onto the sample. The resulting transmitted intensity distribution was acquired using a Thorlabs MVL6X3Z microscope (maximum magnification of 18X) projecting the image onto a DCC1545M CMOS camera. A typical view of a sample slice with the corresponding transmission cross-section is presented in Fig.2. 


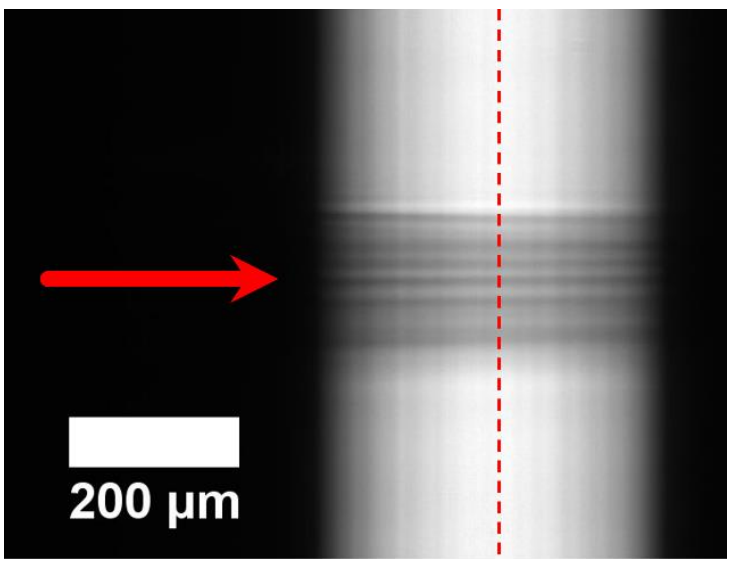

a

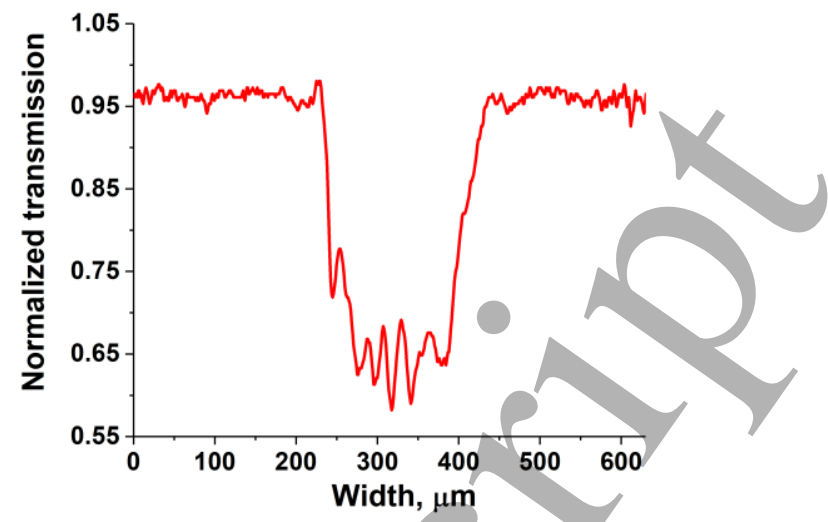

b

Figure 2. (a) Typical view of the darkening observed at a propagation depth of about $1 \mathrm{~mm}$. The direction of laser beam propagation is denoted by the arrow. A cross-section of the transmission data along the dotted line is shown in (b) for a wavelength of $488 \mathrm{~nm}$. Generation of the color centers was performed using the Ti:Sapphire laser, wavelength of $800 \mathrm{~nm}$, pulse duration of $140 \mathrm{fs}$, average power of $540 \mathrm{~mW}, 180$ pulses per point (repetition rate of $1 \mathrm{kHz}$ ), $125 \mu \mathrm{m}$ laser spot diameter. The geometric focus was located at a depth of $3.7 \mathrm{~mm}$

As one can see, this method allowed spatial mapping of the color centers over relatively large distances (up to the sample size) with resolution sufficient to observe the fine features inside of the beam area that appeared due to the multiple filamentation [36]. Two-dimensional transmission maps resulting from the concatenation of the cross-sections acquired in this way are presented in the Results and Discussion section of this work.

\section{Results and Discussion}

\subsection{Optical properties}

There exists a wide range of experimental parameters that lead to the color center generation in borosilicate glass. Most of them lie below the damage threshold of the glass, as higher laser intensities result in plasma shielding, which prevents the laser radiation from being efficiently absorbed by the medium $[37,38]$.

Typical transmission spectra produced as a result of femtosecond laser irradiation of a Borofloat 33 glass sample are shown in Fig. 3. The laser average power values were varied from the one corresponding to threshold for color center generation to damage point of the glass. 


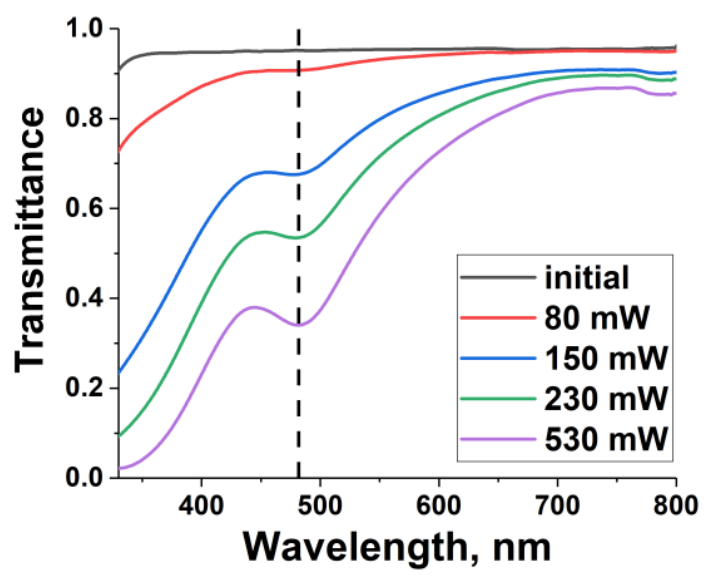

Figure 3. Transmission spectra of color centers produced at different laser average power values. The dashed line denotes the peak absorption wavelength of boron-oxygen hole centers (BOHC) at approximately $490 \mathrm{~nm}$.

As one can see, laser irradiation over a wide range of the average power values results in a decrease in transmission for the UV part of spectrum (wavelengths of around 300-400 nm). Moreover, a distinct transmission minimum can be observed at a wavelength of around 490 $\mathrm{nm}$. The former feature is typically ascribed to silicon- and oxygen-related color centers [34]. It should be mentioned here that similar silicon-related color centers (including the ones caused by electron and hole traps) were also observed in other types of glasses, e.g. soda-lime and other sodium-silicate glasses [38-40]. The $490 \mathrm{~nm}$ minimum, in turn, is a distinctive feature of boron-containing glasses, as it is usually ascribed to boron-oxygen hole centers (BOHC) $[34,35,41]$. The loss of blue light in transmission gave the laser irradiated volume a brown color when observed by the naked eye.

In the following part of this work, close attention will be paid to the BOHC color centers due to the fact that the investigated phenomena are characteristic for the borosilicate glass and were not observed for other glass types [27].

\subsection{Relation between optical properties and HF reactivity}

Using the setup described in the Experimental section, two-dimensional maps of the change in transmission of the sample at a wavelength of $488 \mathrm{~nm}$ were measured. The characteristic absorption wavelength of the BOHC color centers is approximately $490 \mathrm{~nm}$, so the transmission change close to this wavelength was studied as $488 \mathrm{~nm}$ bandpass filters are readily available for applications involving the argon ion laser. A typical result of such a study is presented in Fig. 4 a. 


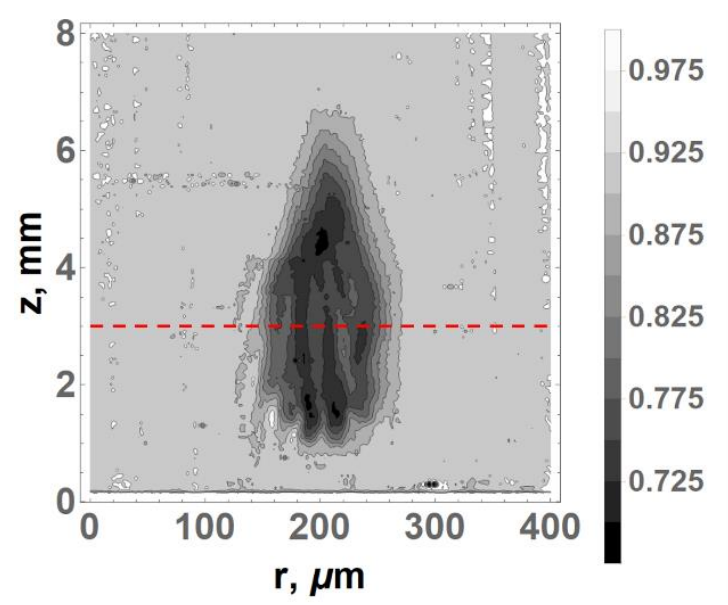

a

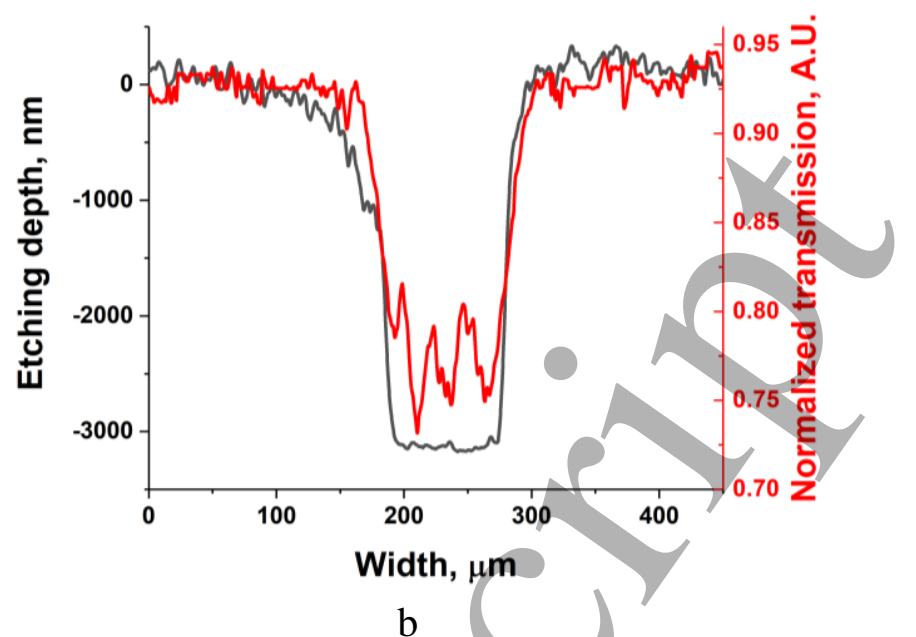

Figure 4. (a) Two-dimensional transmission map (at the wavelength of $488 \mathrm{~nm}$ ) of a sample irradiated at an average power of $90 \mathrm{~mW}$. A z-value of $0 \mathrm{~mm}$ corresponds to the front surface of the sample, where the converging laser beam entered. The dashed line denotes the depth at which the transmission cross-section shown in (b) was recorded. An HF-etched profile of the back surface of a $3 \mathrm{~mm}$ thick sample irradiated at similar conditions (black line) is also shown in (b).

It can be seen from Fig.4a that the distribution of the transmission change due to the color centers inside of glass is quite complicated. This is interpreted to be defined by the laser beam propagation, which in turn is governed by three main processes: Kerr self-focusing, plasma defocusing, and multiple filamentation of the beam caused by the medium inhomogeneities [36]. The interplay of these phenomena can lead to the self-channelling observed between the depths of 1 and $3.5 \mathrm{~mm}$. As well as the non-linear processes the initial curvature of the wavefront due to the presence of the focussing lens results in the beam converging to a minimum size at around its geometric focus (depth of $5 \mathrm{~mm}$ ). Here, the irradiance was at a maximum and the color centers formed have a corresponding minimum optical transmission.

According to the results shown in Fig. $4 \mathrm{~b}$, there exists a certain correlation between the change in transmission at the color center wavelength and change in HF etching rate of the glass sample. As one can see, the cross-section of the transmission map taken at the depth of $3 \mathrm{~mm}$ closely follows the etched profile of the back surface of a $3 \mathrm{~mm}$ thick sample irradiated under similar experimental conditions. Smaller features in the transmission cross-section arising from the multiple filamentation, however, seem to be smoothed out by the intrinsically isotropic character of HF etching process [42] despite the laser-induced anisotropy. By varying the average power of the laser, it is possible to see the effect on the chemical etch depth (Fig 5). There is a soft threshold for the process at around $100 \mathrm{~mW}$ followed by a steep increase in etch rate that correlates with average power as well as number of pulses that each area is exposed to. This effect begins to saturate with increasing power until damage occurs at $>500 \mathrm{~mW}$ and the chemical etch rate is dominated by the surface area presented by microcracks. The average power used in subsequent studies was limited to $<350 \mathrm{~mW}$ to ensure laser-induced damage was avoided. 


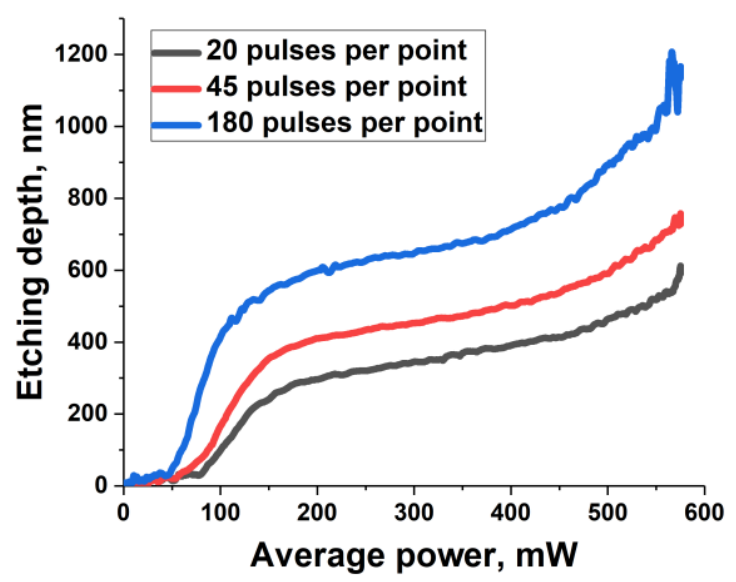

Figure 5. Dependence of chemical etch depth on incident laser average power for three different pulse overlap regimes set by the sample translation velocity.

In order to quantitatively analyze the relationship between the presence of color centers and change in sample HF reactivity, transmission cross-section widths (measured as the width where the optical transmission had halved ) were compared to corresponding widths of etched profiles in a wide range of experimental parameters. Namely, the average power was varied from 10 to $310 \mathrm{~mW}$ for different geometric focus positions. In each case, four values of the propagation depth/sample thickness were studied: 1, 2, 3, and $5 \mathrm{~mm}$. Typical data resulting from the average power variation for fixed thickness and focus position is shown in Fig.6a. These values were used to calculate a correlation coefficient of $\approx 0.997$ between optical transmission and chemical etch profiles. Similar correlation coefficients calculated for different focus positions and sample thicknesses are plotted in Fig.6b.
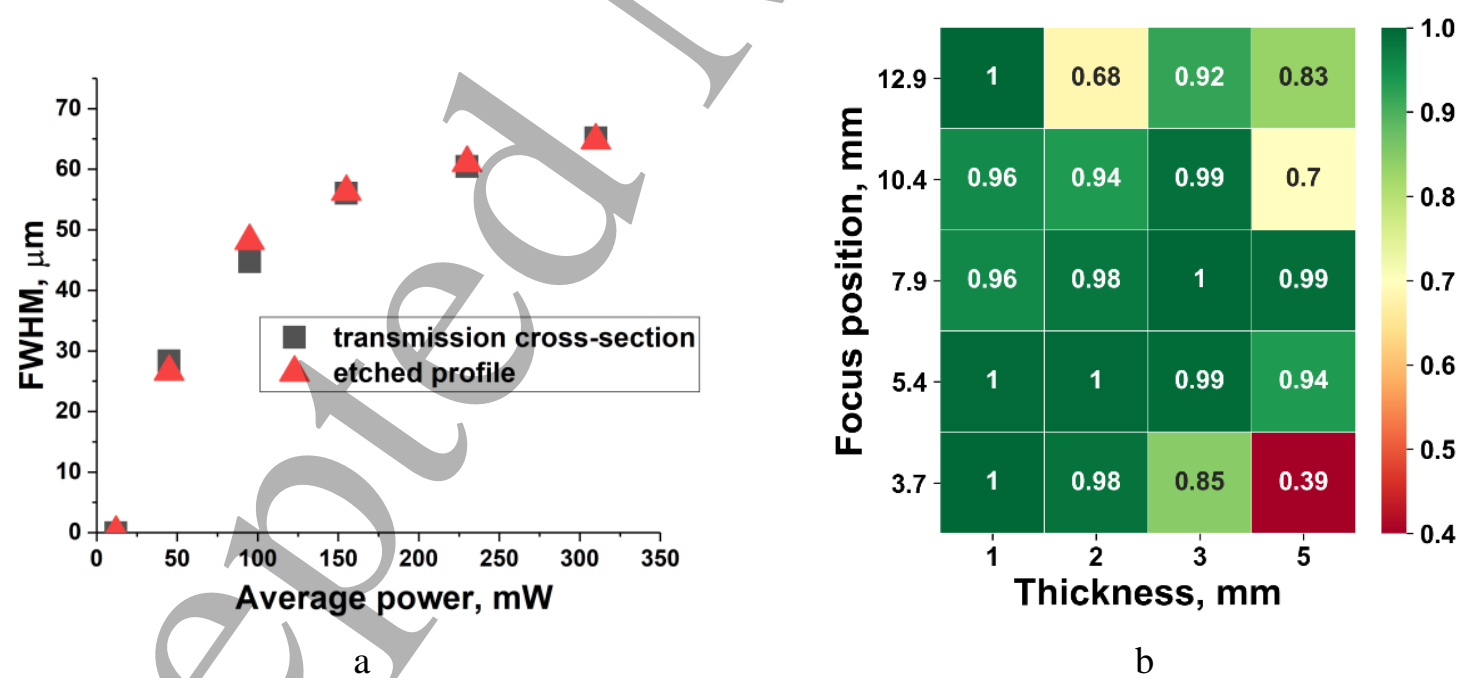

Figure 6. (a) Comparison of the HF-etched profile and transmission cross-section widths (measured as the full width where the transmission had halved) on laser average power. The etched profiles were obtained by measuring the back surface of $1 \mathrm{~mm}$ thick samples. The transmission cross-sections were taken from two-dimensional maps at a propagation depth of $1 \mathrm{~mm}$. (b) Correlation coefficients calculated using data similar to those one shown in (a) for different experimental parameters

According to the results shown in Fig. 6b, the correlation coefficients between the FWHM values of transmission cross-sections and etched profiles remain relatively high (greater than 
0.98) for a wide range of experimental parameters. The values are observed to decrease with the shift of the geometric focus position further away from the sample surface. This fact can be explained by the appearance of laser-induced breakdown in air, which in turn can affect the back-surface properties of the sample. The low correlation value of approximately 0.38 observed for the focus position of $3.7 \mathrm{~mm}$ at the depth of $5 \mathrm{~mm}$ can be accounted for by the beam waist proximity to the back surface of the sample.

The correlation observed here between the appearance of color centers and changes in the HF reactivity of the glass samples is consistent with reports describing the effect to be characteristic for boron-containing-glasses [27,29] and be dependent on the electric charge distribution [28]. In this way, measurement of the laser-induced optical absorption provides a way to predict the resultant acid etch profile. This then allows for a process whereby a "latent image" is written into the glass below the damage threshold which is then revealed as surface relief once "developed" in the acid bath.

One should mention, however, that the exact processes responsible for the accelerated etching provided by color centers are not revealed by these experiments. The preliminary EDX and Raman analyses carried out as a part of this work showed no change in surface properties after laser irradiation. Measuring the optical transmission of the samples during heating showed annealing behaviour similar to that observed in [28]. Based on these observations, one can exclude possible changes in chemical composition and mechanical strain as possible explanations of the altered etching rate. It also demonstrates that the color centers can be removed resulting in a reversible process prior to chemical etching.

\subsection{Surface characterization of the HF etched samples}

The distribution of the color centers within the bulk of the material and, consequently, those at the rear surface, is a complex function of incident laser parameters and the effect that the non-linear response of the material has on the beam propagation. This is not the case for the front surface of the glass where the irradiance pattern is more readily obtained from the linear beam propagation approximation in air with a low level of spherical aberration from the lens. Consequently, this section discusses the roughness of the front surface of the laser irradiated glass where the expectation is that surface structures caused by inhomogeneity of the beam are at a minimum.

According to the results presented above, femtosecond laser irradiation under certain experimental conditions leads to a change in the HF reactivity of glass. It should be noted that such irradiation affects both etching rate and surface roughness. A typical SEM view of the border between the irradiated and unirradiated areas that have both been subjected to HF etching is shown in Fig. 7. 


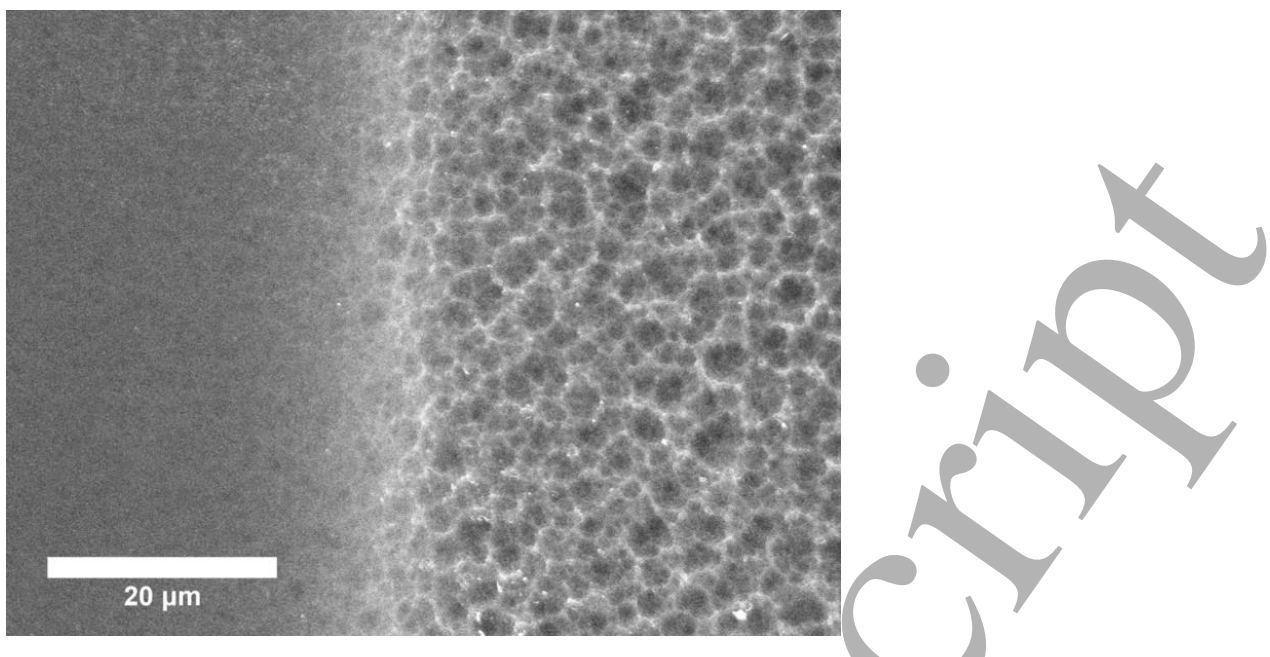

Figure 7. SEM view of a border between the irradiated (left half) and unirradiated (right half) sample areas following HF etching in $10 \%$ solution for 1 hour of the whole area. The laser irradiation conditions were: Ti:Sapphire laser, wavelength of $800 \mathrm{~nm}$, pulse duration of $140 \mathrm{fs}, 180$ pulses per point (repetition rate of $1 \mathrm{kHz}$ ), $125 \mu \mathrm{m}$ laser spot diameter, average power of $230 \mathrm{~mW}$

The irradiated area displays an increased amount of removed material (lower overall surface level), as well as lower roughness as compared to the acid etched unirradiated glass. Further analysis was carried out to quantitatively assess the roughness parameter. The resulting data obtained using a white-light interferometer (WLI) are presented in Fig. 8.

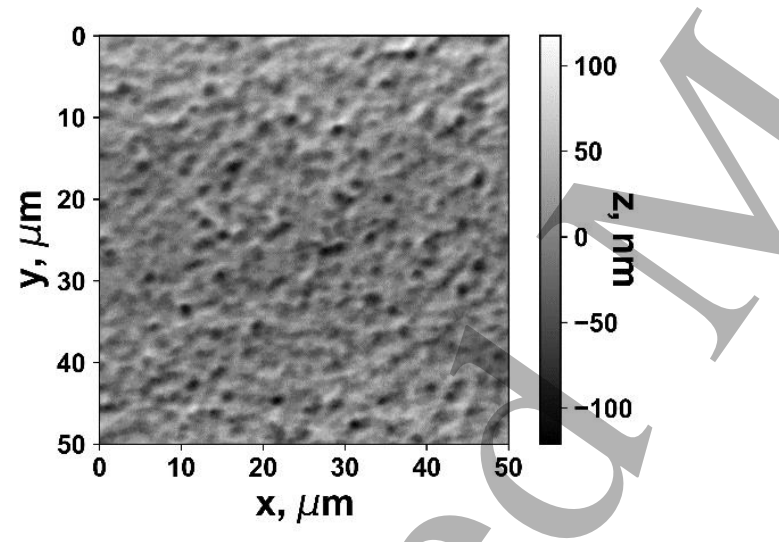

a

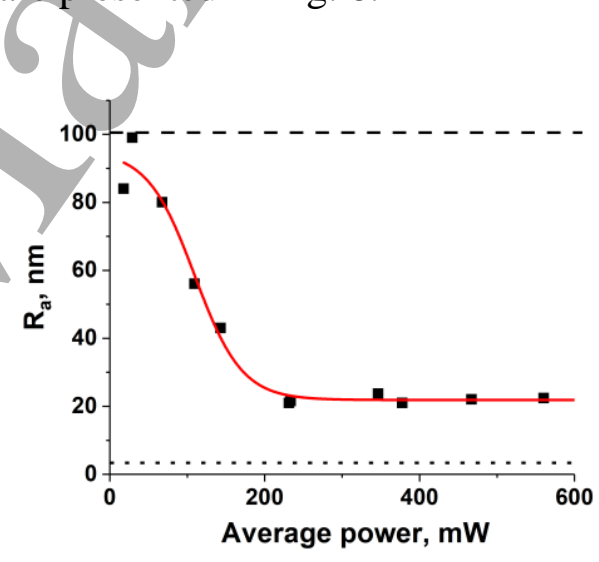

b

Figure 8. (a) Typical view of the irradiated glass surface after HF etching obtained using a white-light interferometer (WLI). (b) Dependence of the front surface average roughness $\left(\mathrm{R}_{\mathrm{a}}\right.$, calculated using the WLI data) on laser average power. Dashed line denotes the surface roughness of a typical unirradiated area after etching; dotted line denotes the surface roughness of the glass in as-received condition prior to HF etching. The same conditions (except for the average power) as in figure 5 were used

It can be seen from both figure 6 (right) and figure $7 \mathrm{~b}$ that unirradiated Borofloat 33 that has been subjected to $\mathrm{HF}$ etching becomes rough $\left(\mathrm{R}_{\mathrm{a}} \sim 100 \mathrm{~nm}\right)$ and scatters light making in unsuitable for optical applications. However, increasing the density of color centers through applying larger average power decreases the surface roughness to levels that are "glossy" to the unaided eye with low degrees of scattering. This effect saturates after $\sim 230 \mathrm{~mW}$ at a value of $R_{a} \sim 20 \mathrm{~nm}$ which is acceptable for certain types of optical elements albeit higher than the as-received surface. 


\subsection{Possible applications}

By obtaining the dependence of the etching depth on laser parameters, it is possible to create a surface with the desired relief profile. However, there exist certain restrictions to the topography that can be realised. First of all, the maximum etching depth that can be achieved by this method typically does not exceed several micrometres. Secondly, the resolution in the surface plane is limited by the finite laser spot size on the surface and the fact that HF is an inherently isotropic process [42]. Namely, even if one creates anisotropy by laser irradiation, the etching process will still happen, although more slowly, in all the other directions not defined by it.

Taking into account these restrictions, the fabrication of relatively shallow surfaces with slowly varying relief has been investigated. One of the examples of such a surface is a socalled Laplacian window [43]. Such an optical element has a freeform surface, whose effect on the light passing thorough it is defined by the value of the Laplace operator of its surface relief. In order to calculate the surface relief reproducing the desired intensity distribution one thus needs to solve the Poisson's equation [43-45].

The relatively simple intensity pattern of a ring was chosen for a test window. By solving the Poisson's equation in the manner given in [43-45], one can calculate the corresponding surface relief (Fig. 9a). It should be noted here that the absolute values of the surface depth do not play an important role; it is the aspect ratio of $\mathrm{X}-\mathrm{Y}$ size to depth that defines the local radius of curvature and hence whether the Laplacian condition is fulfilled [43,44]. Here, if the etching depth that can be achieved by this method is of the order of several micrometres, the linear dimensions of the window will be of several millimetres. The comparison of the crosssections of the theoretical relief and experimentally fabricated window is shown in Fig. 9b.

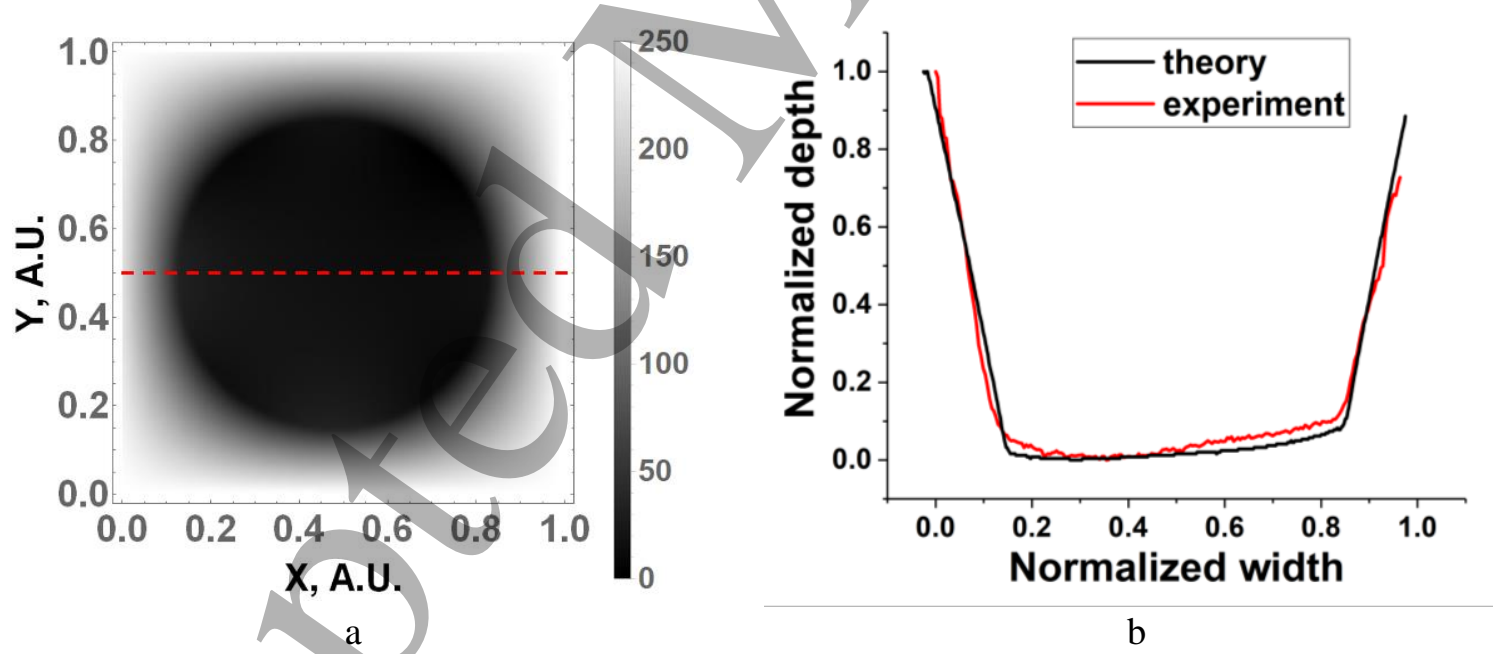

Figure 9. (a) Theoretical profile of a test Laplacian window. (b) Comparison of the theoretical crosssection taken along the dashed line in (a) with the experimental profile. Note that the real size of the test Laplacian window was of $2.5 \times 2.5 \mathrm{~mm}$ with the maximum depth of $\sim 1 \mu \mathrm{m}$

As it can be seen, the method results in good reproduction of the theoretical relief with an average deviation with respect to the total depth of $\sim 3 \%$. It should be noted that the absolute value of the depth is of about $1 \mu \mathrm{m}$, so the discrepancy with the theoretical profile is typically of the order of tens of nanometres. 
The performance of the resulting window was assessed by illuminating it with a collimated light source and recording the projected intensity using a CCD camera. The resulting intensity distributions are shown in Fig. 10.

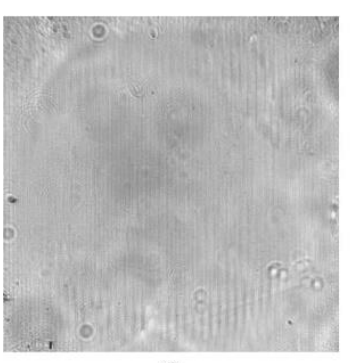

a

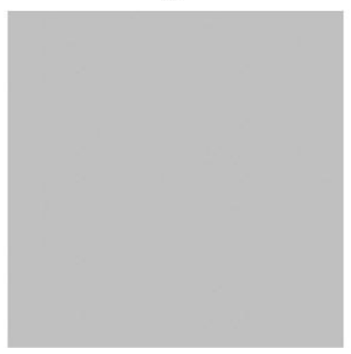

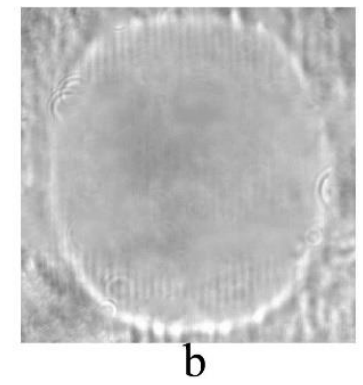

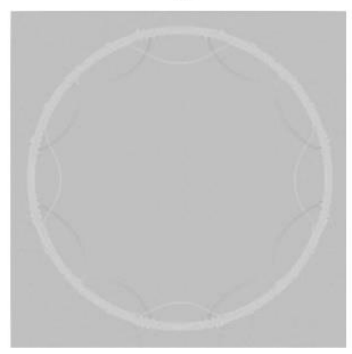

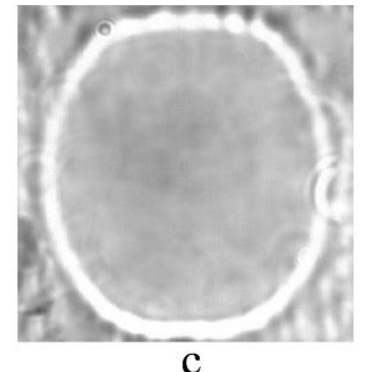

c

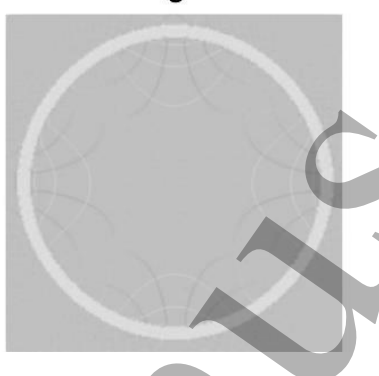

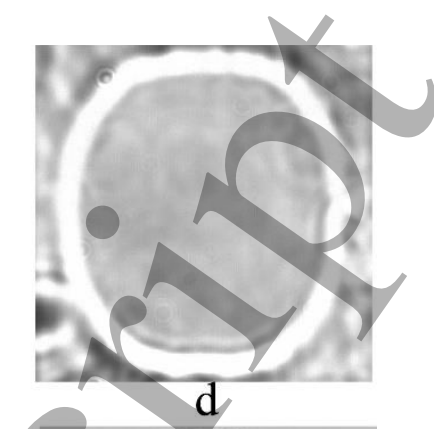

d

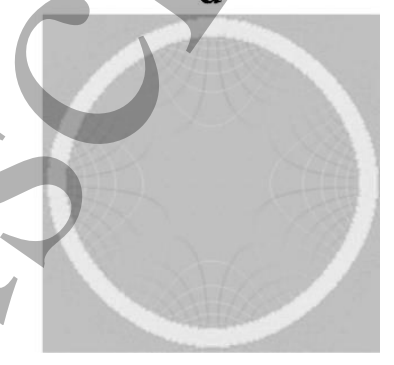

Figure 10. Comparison of the light intensity after the test Laplacian window with the theoretical data at different distances from the designed plane of observation: (a) at the image plane, (b) $-5 \mathrm{~mm}$, (c) $15 \mathrm{~mm}$, (d) $-30 \mathrm{~mm}$. The negative values of the distance account for the CCD camera being closer to the sample than the intended plane

By reference to the images in Fig.10, it can be seen that the method of laser-induced reactivity change has allowed fabrication of a Laplacian window. The intensity distributions qualitatively follow those calculated from the analysis of the refraction through the surface relief. One should also note the residual roughness on the surface of the sample does not significantly affect its performance due to the Laplacian pre-focal brightening being located at a considerable distance from the conventional image plane.

\section{Conclusions}

It has been shown that femtosecond laser irradiation of borosilicate glass can induce the appearance of color centers and that these defects have a higher level of reactivity to hydrofluoric acid than the unirradiated material. The optical transmission characteristics of the darkened glass have allowed the process to be ascribed to boron-oxygen hole centers (BOHC). Thermal annealing can remove these hole traps and so the process is reversible. The optical density map closely correlates to the acid-revealed relief profile via the increase in reactivity. In this way, the reversible laser-induced optical changes act as a tool to predict the final topography of the surface. These findings have been used to demonstrate a process where a desired distribution of color centers can be programmatically laser generated, the pattern checked optically, erased by thermal annealing if required, and then made into a permanent surface relief by exposure to acid. This is a flexible route to fabricate freeform surfaces in borosilicate optical glasses. Utilising this technique, the production of a glass Laplacian window has been demonstrated for the first time.

The spatial distribution of the laser-induced color centers in the bulk of the glass is defined by the propagation of the beam, which in turn is governed by Kerr self-focusing, plasma formation, and multiple filamentation. As the presence of color centers was found to correlate 
with the change of the glass reactivity in HF over a wide range of experimental parameters, the roughness of the etch is influenced by the uniformity of the laser irradiance. However, at the front surface of the glass, where the laser beam profile has not been modulated by nonlinear effects, the surface arithmetic mean deviation $\left(R_{a}\right.$ value) was found to be lower for glass that had been laser irradiated below the ablation threshold than unirradiated regions: after 1 hour of etching in 10\% HF solution, the areas with color centers had about 5 times lower $\mathrm{R}_{\mathrm{a}}$ values than unirradiated ones $(20 \mathrm{~nm}$ versus $100 \mathrm{~nm})$. The reason for this is not readily apparent. Structural studies using XRD and Raman spectroscopy have not revealed any large changes for the irradiated areas and EDX analysis has not shown any preferential loss of species. It is known that the roughening of glass induced by HF etching is worse when insoluble by-products such as $\mathrm{CaF}_{2}, \mathrm{MgF}_{2}$ and $\mathrm{AlF}_{3}$ form [46] which affect the local etch rate. In addition, in "wet" silica (i.e. $\mathrm{SiO}_{2}$ in the presence of $\mathrm{OH}$ groups), laser irradiation can affect the bonding configuration of $\mathrm{SiOH}$ [47] and hence its reactivity. The interplay of these processes is the subject of further study.

Whilst the laser irradiation is serial in nature due to its point-by-point exposure of the glass, the acid etch is a parallel process; the anisotropy required to give the spatially varying depth comes from the laser-induced reactivity change and so the whole sample can be submerged. It may be possible to make the laser exposure also into a one-step method by utilising large format beams in the UV and a grey tone mask in a projection system (e.g. excimer laser systems). This will require verification that UV-induced color centers behave in the same manner as the near IR non-linear processes used here.

\section{Acknowledgements}

This work is sponsored by The Leverhulme Trust as project RPG-2016-181. The authors acknowledge useful discussions with Prof M V Berry and Prof D E Jesson.

\section{References}

[1] Fang F Z, Zhang X D, Weckenmann A, Zhang G X and Evans C 2013 Manufacturing and measurement of freeform optics CIRP Ann. 62 823-46

[2] Bekesi J, Meinertz J, Simon P and Ihlemann J 2013 Sub-500-nm patterning of glass by nanosecond $\{\mathrm{KrF}\}$ excimer laser ablation Appl. Phys. A $11017-21$

[3] Ihlemann J and Wolff-Rottke B 1996 Excimer laser micro machining of inorganic dielectrics Appl. Surf. Sci. 106 282-6

[4] Piontek M C, Herrmann T and L'huillier J A 2017 Selective glass surface modification with picosecond laser pulses for spatially resolved gloss reduction $J$. Laser Appl. 2922507

[5] Herman P R, Oettl A, Chen K P and Marjoribanks R S 1999 Laser micromachining of transparent fused silica with 1-ps pulses and pulse trains ed M K Reed and J Neev (San Jose, CA) pp 148-55

[6] Ben-Yakar A and Byer R L 2004 Femtosecond laser ablation properties of borosilicate glass J. Appl. Phys. 96 5316-23

[7] Ozkan A, Migliore L R, Dunsky C M and Phaneuf M W 2003 Glass processing using microsecond, nanosecond and femtosecond pulsed lasers Fourth International Symposium on Laser Precision Microfabrication vol 5063 (International Society for 
Optics and Photonics) pp 108-13

[8] Nowak K M, Baker H J and Hall D R 2006 Efficient laser polishing of silica microoptic components Appl. Opt. 45 162-71

[9] Wlodarczyk K L, Mendez E, Baker H J, McBride R and Hall D R 2010 Laser smoothing of binary gratings and multilevel etched structures in fused silica Appl. Opt. 49 1997-2005

[10] Wang J, Niino H and Yabe A 1999 One-step microfabrication of fused silica by laser ablation of an organic solution Appl. Phys. A Mater. Sci. Process. 68 111-3

[11] Ding X, Kawaguchi Y, Sato T, Narazaki A, Kurosaki R and Niino H/2004 Micronand submicron-sized surface patterning of silica glass by LIBWE method $J$.

Photochem. Photobiol. A Chem. 166 129-33

[12] Huang Z Q, Hong M H, Tiaw K S and Lin Q Y 2007 „Quality glass processing by Laser Induced Backside Wet Etching” JLMN-Journal of Laser Micro/Nanoengineering 2 194-9

[13] Kopitkovas G, Lippert T, David C, Wokaun A and Gobrecht J 2003 Fabrication of micro-optical elements in quartz by laser induced backside wet etching Microelectron. Eng. 67 438-44

[14] Zimmer K and Böhme R 2005 Precise etching of fused silica for micro-optical applications Appl. Surf. Sci. 243 415-20

[15] Tsvetkov M Y, Yusupov V I, Minaev N V, Timashev P S, Golant K M and Bagratashvili V N 2016 Effects of thermo-plasmonics on laser-induced backside wet etching of silicate glass Laser Phys. Lett. 13106001

[16] Tsvetkov M Y, Yusupov V I, Minaev N V, Akovantseva A A, Timashev P S, Golant K M, Chichkov B N and Bagratashvili V N 2017 On the mechanisms of single-pulse laser-induced backside wet etching Opt. Laser Technol. 88 17-23

[17] Zimmer K and Böhme R 2008 Laser-induced backside wet etching of transparent materials with organic and metallic absorbers Laser Chem. 2008

[18] Marcinkevičius A, Juodkazis S, Watanabe M, Miwa M, Matsuo S, Misawa H and Nishii J 2001 Femtosecond laser-assisted three-dimensional microfabrication in silica Opt. Lett. $26277-9$

[19] Cheng Y, Sugioka K and Midorikawa K 2004 Microfluidic laser embedded in glass by three-dimensional femtosecond laser microprocessing Opt. Lett. 29 2007-9

[20] Matsuo S, Sumi H, Kiyama S, Tomita T and Hashimoto S 2009 Femtosecond laserassisted etching of Pyrex glass with aqueous solution of KOH Appl. Surf. Sci. 255 9758-60

[21] Wu D, Wu S, Xu J, Niu L, Midorikawa K and Sugioka K 2014 Hybrid femtosecond laser microfabrication to achieve true 3D glass/polymer composite biochips with multiscale features and high performance: the concept of ship-in-a-bottle biochip Laser Photon. Rev. 8 458-67

[22] Chen F, Deng Z, Yang Q, Bian H, Du G, Si J and Hou X 2014 Rapid fabrication of a large-area close-packed quasi-periodic microlens array on BK7 glass Opt. Lett. 39 
$606-9$

[23] Tsai H-Y, Luo S-W and Chang T-L 2015 Surface forming on glass material by femtosecond laser modification with HF etching process CIRP Ann. 64 205-8

[24] Wang M R and Su H 1998 Laser direct-write gray-level mask and one-step etching for diffractive microlens fabrication Appl. Opt. 37 7568-76

[25] Wang M R and Su H 1998 Multilevel diffractive microlens fabrication by one-step laser-assisted chemical etching upon high-energy-beam sensitive glass Opt. Lett. 23 $876-8$

[26] Kim J, Berberoglu H and Xu X 2004 Fabrication of microstructures in photoetchable glass ceramics using excimer and femtosecond lasers J. Micro/Nanolithography, MEMS, MOEMS 3 478-86

[27] Sauvain E, Kyung J H and Lawandy N M 1995 Multiphoton-micrometer-scale photoetching in silicate-based glasses Opt. Lett. 20 243-5

[28] Kyung J H and Lawandy N M 1996 Maskless photoencoded selective etching for glass-based microtechnology applications Opt. Lett. 21 174-6

[29] Glebov L B, Glebova L and Lopatiuk O 2004 Photoinduced chemical etching of silicate and borosilicate glasses Glas. Sci. Technol. AM MAIN- 75 298-301

[30] SCHOTT Technical Glass Solutions GmbH 2009 Schott Borofloat 33

[31] Mackey J H, Smith H L and Halperin A 1966 Optical studies in X-irradiated high purity sodium silicate glasses J. Phys. Chem. Solids 27 1759-72

[32] Barker R S, Richardson D A, McConkey E A G and Yeadon R E 1960 Radiationinduced defects in lead silicate glass Nature 1881181

[33] Bishay A 1970 Radiation induced color centers in multicomponent glasses J. Non. Cryst. Solids 3 54-114

[34] Ehrt D and Ebeling P 2003 Radiation defects in borosilicate glasses Glas. Technol. 44 46-9

[35] Griscom D L, Sigel Jr G H and Ginther R J 1976 Defect centers in a pure-silica-core borosilicate-clad optical fiber: ESR studies J. Appl. Phys. 47 960-7

[36] Couairon A and Mysyrowicz A 2007 Femtosecond filamentation in transparent media Phys. Rep. 441 47-189

[37] Stuart B C, Feit M D, Herman S, Rubenchik A M, Shore B W and Perry M D 1996 Nanosecond-to-femtosecond laser-induced breakdown in dielectrics Phys. Rev. B 53 1749

[38] Efimov O M, Gabel K, Garnov S V, Glebov L B, Grantham S, Richardson M and Soileau M J 1998 Color-center generation in silicate glasses exposed to infrared femtosecond pulses JOSA B 15 193-9

[39] Lonzaga J B, Avanesyan S M, Langford S C and Dickinson J T 2003 Color center formation in soda-lime glass with femtosecond laser pulses J. Appl. Phys. 94 4332-40

[40] Efimov O M, Glebov L B, Grantham S and Richardson M 1999 Photoionization of silicate glasses exposed to IR femtosecond pulses J. Non. Cryst. Solids 253 58-67 
[41] White W T, Henesian M A and Weber M J 1985 Photothermal-lensing measurements of two-photon absorption and two-photon-induced color centers in borosilicate glasses at $532 \mathrm{~nm}$ JOSA B 2 1402-8

[42] Spierings G 1993 Wet chemical etching of silicate glasses in hydrofluoric acid based solutions J. Mater. Sci. 28 6261-73

[43] Berry M V 2017 Laplacian magic windows J. Opt. 19 06LT01

[44] Berry M V 2005 Oriental magic mirrors and the Laplacian image Eur. J. Phys. 27109

[45] Bawart M, Bernet S and Ritsch-Marte M 2017 Programmable freeform optical elements Opt. Express 25 4898-906

[46] Iliescu C, Chen B and Miao J 2008 On the wet etching of Pyrex glass Sensors Actuators, A Phys. 143 154-61

[47] Hosono H, Mizuguchi M, Kawazoe H and Ogawa T 1999 Effects of fluorine dimer excimer laser radiation on the optical transmission and defect formation of various types of synthetic $\mathrm{SiO}_{2}$ glasses Appl. Phys. Lett. $742755-7$ 